\title{
La asistencia urológica en el Centro Sanitario Integral "Alto Palancia": modelo de integración Primaria-Especializada y Consulta Única
}

\author{
J.A. Queipo Zaragozá, A. Moral Baltuille*, J.F. Beltrán Meseguer, A. Marco Aznar**, \\ A. Borrell Palanca, F. Chicote Pérez, E. Armañanzas Villena***, F. Pastor Sempere
Servicio de Urología, Hospital de Sagunto, Valencia. *Subdirectora Médica. Hospital de Sagunto. Valencia. **Unidad de Absentismo de Funcionarios de la Generalitat Valenciana. Valencia. ***Director. Hospital de Sagunto. Valencia.

Actas Urol Esp 2005; 29 (3): 305-310

\begin{abstract}
RESUMEN
LA ASISTENCIA UROLÓGICA EN EL CENTRO SANITARIO INTEGRAL “ALTO PALANCIA": MODELO DE INTEGRACIÓN PRIMARIA-ESPECIALIZADA Y CONSULTA ÚNICA

Introducción y objetivos: El progresivo incremento en la demanda de asistencia urológica exige establecer nuevos modelos asistenciales que eviten la saturación de consultas y mejoren la calidad en la atención al usuario. El objetivo de este trabajo es analizar la mejora de la actividad asistencial desarrollada en el Centro Sanitario Integral "Alto Palancia", tras la instauración de nuevos modelos asistenciales basados en la integración entre Atención Primaria y Especializada y la puesta en práctica de la denominada Consulta Única.

Material y métodos: Analizamos las características del Centro, funciones y actividad de la especialidad desarrollada durante el año 2002. Describimos la aplicación del modelo de Consulta Única y los elementos de integración con Atención Primaria. Analizamos la mejora de la actividad a través de indicadores, que comparamos con años precedentes. Para determinar el grado de satisfacción de los usuarios realizamos una encuesta de opinión de pacientes y/o familiares atendidos en el Centro bajo los nuevos modelos asistenciales. Finalmente analizamos las ventajas que el proceso aplicado presenta para el paciente y para la propia institución sanitaria.

Resultados: $\mathrm{El} 42 \%$ de pacientes han sido atendidos mediante el modelo de Consulta Única, siendo la patología predominante la HBP en el varón y las ITUs en la mujer. El tiempo de demora para primera visita se ha reducido desde 49 días en diciembre 2001 a solo 3 en diciembre 2002. Solo el 7,7\% de pacientes dados de alta para seguimiento por Atención Primaria han sido remitidos de nuevo al especialista. El tiempo que el urólogo dedica a consultas se ha reducido en un $29 \%$, siendo este tiempo dedicado a la realización cirugía menor y exploraciones complementarias (ecografias, estudios urodinámicos, etc.). La encuesta de opinión ha puesto de manifiesto una satisfacción del usuario muy elevada.

Conclusiones: El modelo de Atención Integral Primaria-Especializada y la puesta en práctica de la Consulta Única son aplicables a la especialidad de Urología, incrementando la calidad percibida por el usuario y con evidentes ventajas para la Organización Sanitaria.

Palabras clave: Asistencia Integral. Consulta Única. Calidad Percibida.
\end{abstract}

\section{ABSTRACT}

UROLOGICAL ATTENDANCE IN INTEGRAL CENTER SANITARI "ALTO PALANCIA": MODEL OF PRIMARY-SPECIALIZED INTEGRATION AND UNIQUE CONSULTATION

Introduction and objective: The progressive increase in the demand of urological attendance demands to establish new welfare models that avoid the saturation of consultations and improve the quality in the attention to the user. The objective of this work is to analyze the improvement of the welfare activity developed in Integral the Sanitary Center Alto Palancia, after the restoration of new welfare models based on integration between Attention Primary and Specialized and the putting in practice of the denominated Unique Consultation.

Material and methods: We analyzed the characteristics of the Center, functions and activity of the specialty developed during year 2002. We described to the application of the model of Unique Consultation and the elements of integration with Primary Attention. We analyzed the improvement of the activity through indicators, that we compared with preceding years. In order to determine the degree of satisfaction of the users we made a survey of opinion of patients and/or taken care of relatives in the Center under the new welfare models. Finally we analyzed the advantages that the applied process presents for the patient and the own sanitary institution.

Results: $42 \%$ of patients have been taken care of by means of the model of Unique Consultation, being the predominant pathology the HBP in the man and the ITUs in the woman. The time of delay for first visit has been reduced from 49 days in single December 2001 to 3 in December 2002. Single $7.7 \%$ of patients discharged from the hospital for pursuit by Primary Attention have been sent again to the specialist. The time that the urólogo dedicates to consultations has reduced in a $29 \%$, being this time dedicated to the accomplishment smaller surgery and final reconnaissances (echography, urodynamic studies and others). The opinion survey has shown a satisfaction of the user very elevated.

Conclusion: The model of Integral Attention Primary-Specialized and the putting in practice of Unique Consultation applicable to the specialty of Urology, increasing the quality perceived by the user and with evident advantages for the Sanitary Organization.

Keywords: Integral Attendance. Unique Consultation. Perceived Quality. 


\section{INTRODUCCIÓN Y OBJETIVOS}

El progresivo incremento en la demanda asistencial especializada obliga a crear nuevos Centros, más próximos al usuario, con modelos asistenciales que potencien el papel de la Atención Primaria y establezcan una consulta más funcional, que evite las consultas saturadas. El Centro Sanitario Integral "Alto Palancia", de reciente creación, está situado en la localidad de Segorbe; da asistencia especializada a la Comarca del "Alto Palancia", emplazada en el interior de la provincia de Castellón. Esta comarca abarca un total de 26 pequeños municipios con una baja y muy dispersa densidad poblacional (22,3 habitantes $/ \mathrm{Km}^{2}$ ). En el mismo Centro se lleva a cabo la Atención Primaria y Especilizada. El hecho de compartir el mismo espacio físico ha permitido establecer una mayor colaboración entre Primaria y Especializada, una actividad asistencial "sin escalones" basada fundamentalmente en la instauración de las denominadas "Consultas de Derivación Dirigida" (CDD) y los "Planes de Seguimiento" por Atención Primaria. El proceso asistencial especializado se desarrolla bajo el modelo de "Consulta Única", que permite establecer un diagnóstico y una estrategia terapéutica en un único tiempo asistencial, evitando así que el usuario deba de acudir repetidas veces al Centro para la resolución de su proceso patológico. Con la CDD el paciente es remitido al especialista con un diagnóstico de presunción al que acompaña una serie de exploraciones básicas que permitirán aplicar el modelo de Consulta Única. Una vez el especialista establece el tratamiento correspondiente, el paciente es remitido de nuevo a Atención Primaria con un Plan de Seguimiento. El hospital de referencia se constituye únicamente como un elemento de apoyo para la realización de exploraciones complementarias de mayor complejidad técnica o para cirugía mayor.

El objetivo de este trabajo es analizar los resultados y las ventajas de la aplicación de estos nuevos modelos asistenciales en la especialidad de Urología en el Centro Sanitario Integral (C.S.I.) "Alto Palancia".

\section{MATERIAL Y MÉTODOS}

Analizamos las características del Centro, las funciones del especialista, cartera de servicios, recursos necesarios y la particular estructuración funcional en base al modelo innovador de "Consulta Única". Comparamos la actividad desarrollada durante el año 2002, año en que se instauran los nuevos modelos asistenciales, y la comparamos con la del 2001. Así mismo, describimos los mecanismos de integración con Atención Primaria y con el Hospital de Referencia, de quien dependemos administrativamente. Para determinar el grado de satisfacción de los usuarios realizamos una encuesta de opinión de pacientes y/o familiares atendidos en el Centro bajo este modelo asistencial. Finalmente analizamos las ventajas que el proceso aplicado presenta para el paciente y para la propia institución sanitaria.

El Centro presenta una estructura arquitectónica horizontal que facilita la accesibilidad del personal y pacientes a las consultas y unidades diagnósticas. Junto a las consultas se han habilitado salas adyacentes para la realización de pruebas diagnósticas. En urología disponemos de un ecógrafo, una camilla y una mesa de exploración, con instrumental propio de la especialidad. La consulta está informatizada, pero manteniendo la historia clínica tradicional. Mediante una red intranet podemos consultar informes de hospitalización o exploraciones complementarias realizadas en el Hospital de Sagunto.

Entre las funciones desarrolladas por el especialista, la actividad asistencial es la predominante; comprende además de la atención de primeras visitas y sucesivas, la realización de exploraciones complementarias como ecografias, estudios urodinámicos, instrumentación propia de la especialidad, etc, que al realizarse en la propia consulta reduce el número de visitas necesarias para establecer un diagnóstico. Al Centro acuden regularmente $2 \mathrm{FEA}$ de Urología, un día a la semana cada uno de ellos. El número de pacientes atendidos al día es de 25, 10 primeras visitas y 15 sucesivas, con un tiempo medio asignado a cada paciente de 20 y 15 minutos respectivamente. Además, dos días al mes se realiza la cirugía menor que en el propio Centro se genera, y que es programada ya en la propia consulta por el especialista y el paciente. Además de la tarea asistencial, es de especial relevancia la actividad formativa dirigida a los facultativos de Atención 
Primaria, lo que permite establecer las bases para la asistencia integral Primaria-Especializada.

Además de la especialidad de Urologia, en el Centro se desarrollan otras 15 especialidades, tanto médicas como quirúrgicas, que progresivamente han ido incorporando a su modus operandi estos modelos asistenciales.

La encuesta de opinión se realizó a partir de una muestra de 145 pacientes y/o familiares atendidos mediante el proceso asistencial de “Consulta Única”. Se solicitaba valorar de 0 a 10 (0 si total insatisfacción y 10 si total satisfacción), una serie de 3 items: 1.- La atención recibida por el especialista y enfermería, 2.- Los medios tecnológicos aplicados en el Centro y 3.- La rapidez en la resolución de su problema. Además se realizaba una pregunta final con posibilidad de respuesta de SI ó NO: ¿Volvería a someterse al proceso asistencial de Consulta Única?

\section{RESULTADOS}

Durante el pasado año 2002 hemos atendido un total de 1976 consultas, 749 primeras visitas y 1227 sucesivas, con índice de frecuentación de 1,64. El 92\% de primeras visitas fueron remitidas desde Atención Primaria y el resto desde otras especialidades como Ginecologia, Cirugía General, Digestivo, Oncología, etc. El número de pacientes atendidos mediante Consulta Única ha sido de 311 (42\%) siendo la patología predominante la HBP en el varón y las infecciones urinarias en la mujer. Se han realizado un total de 786 estudios ecográficos y 64 intervenciones quirúrgicas, con un índice de utilización de quirófano del $82 \%$. El tiempo de demora para $1^{\underline{a}}$ visita se ha reducido desde las 7 semanas en diciembre del 2001 a ser prácticamente inexistente (3 días) en diciembre del 2002. El tiempo de demora para cirugía ambulatoria en el Centro no supera los 28 días. No se ha generado ninguna reclamación. De los 193 pacientes dados de alta con un Plan de Seguimiento para Atención Primaria, solo el 7,7\% han sido remitidos de nuevo al especialista. La aplicación del modelo de Consulta Única permitió reducir en un 29\% el tiempo que el especialista dedica a consultas, respecto del año 2001. Este tiempo fue designado a intervenciones quirúrgicas ambulatorias en el propio Centro, práctica que en el año anterior no se llevaba a cabo. Comparado con el año 2001, último año en el que se siguió el modelo de asistencia tradicional, en el Hospital de Sagunto ha descendido el volumen de $1^{\text {as }}$ visitas en un $18 \%$ y prácticamente ha desaparecido la lista de espera para cirugía menor.

La encuesta de opinión puso de manifiesto un elevado nivel de satisfacción, con puntuaciones medias de 8,9 para el item correspondiente a "La atención recibida por el especialista y enfermería", 8,4 para "Los medios tecnológicos aplicados en el Centro" y 9,2 para "La rapidez en la resolución de su problema”. Sólo un 7\% (10 pacientes) manifestaron no volver a someterse al proceso asistencial de Consulta Única.

\section{DISCUSIÓN}

Integración Atención Primaria-Especializada

Uno de los inconvenientes de nuestro sistema sanitario es el funcionamiento de los diferentes niveles asistenciales en compartimentos estancos, lo que conlleva con frecuencia problemas de coordinación y colaboración entre ellos. Como consecuencia aparecen discrepancias en las actitudes diagnósticas y terapéuticas, que incrementan al final la ineficiencia del sistema y el consumo de recursos ${ }^{1}$. En nuestro Centro, favorecido por la proximidad física entre Atención Primaria y Atención Especializada, elaboramos un plan para establecer una "asistencia sin escalones", donde el profesional de Atención Primaria se hiciera partícipe de la Asistencia Especializada. Uno de los objetivos era evitar el problema que aquejan muchos especialistas de recibir pacientes por procesos de escasa complejidad o sin excesivo criterio, lo que provocaba una sobrecarga y demora en las consultas. El proyecto exigía un intercambio de conocimientos y el establecimiento de actitudes que mejoraran la resolución de los problemas de salud en el menor tiempo posible, incrementando así la calidad percibida por el usuario; en definitiva un trabajo en equipo entre niveles asistenciales. El primer logro del Centro, ya desde su creación, fue ubicarlos en un mismo espacio físico (en el mismo edificio), lo que permite establecer un contacto íntimo entre Atención Primaria y Especializada. La actividad del especialista no se limita únicamente a atender las visitas programadas; su labor Formativa 
constituye el pilar básico de la colaboración entre los niveles asistenciales, desarrollándose periódicamente sesiones clínicas y Guías Clínicas de Actuación. Ello permite, cuando un paciente es remitido al especialista, realizar las denominadas Consultas de Derivación Dirigida (CDD). Experiencias de colaboración entre Primaria y Especializada ya han demostrado altos grados de satisfacción de médicos y pacientes ${ }^{2}$.

\section{Modelo asistencial de "Consulta Única"}

Gracias a la colaboración entre Primaria y Especializada, cuando el paciente es remitido a la consulta, lo es con un diagnóstico de presunción y una serie de exploraciones complementarias básicas a las que el generalista tiene acceso. De este modo, cuando el paciente acude a la primera visita del especialista aporta unos datos que, junto a las exploraciones que se realizan en la consulta o en las Unidades de Apoyo al Diagnóstico, permiten establecer un diagnóstico definitivo y una pauta de tratamiento. La innovación del modelo reside en que las exploraciones que el especialista requiere se realizan en ese primer tiempo asistencial y por tanto se llega en un único proceso al diagnóstico y estrategia terapéutica. Se puede realizar una segunda visita para control del tratamiento. Véase en esquema este modelo (Fig. 1). Una vez establecido el plan terapéutico idóneo, el paciente es remitido a su medico de cabecera con un informe donde se establece el Plan de Seguimiento y los supuestos en los que se deberá remitir de nuevo al especialista.

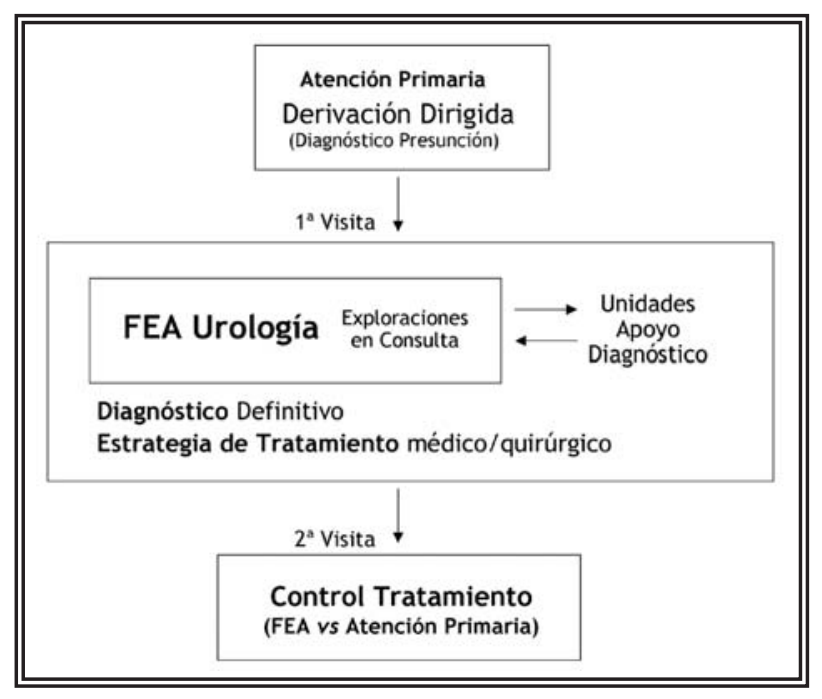

FIGURA 1. Modelo asistencial de "Consulta Única"
La aplicación de este modelo exige la utilización, ya desde Atención Primaria, de las Guías de Práctica Clínica y la puesta en marcha de las denominadas Vías Clínicas. Por Guías de Práctica Clínica se entiende a aquellas recomendaciones diseñadas sobre la base de la evidencia científica que ayudan a los profesionales a elegir la mejor alternativa asistencial. Las Vías Clínicas consistirian en una batería predeterminada de actividades para una asistencia supuesta, que permiten la máxima eficiencia en el proceso asistencial. En Urologia no hemos encontrado excesivos inconvenientes en la aplicación de las Vías Clínicas. En nuestro Centro hemos aplicado estos modelos de forma experimental, basándonos en los procedimientos aplicados en el Hospital "Alto Guadalquivir"3,4.

\section{Vias de integración con el Hospital de}

\section{Referencia}

El Hospital de Sagunto, como Hospital de Referencia, gestiona la asistencia especializada del Centro y se constituye como elemento de apoyo a través de los siguientes aspectos:

1. Los facultativos que acuden al Centro, ejercen el resto de su actividad en el Servicio de Urologia del Hospital de Sagunto. Por ello, cuando el FEA se encuentra ante casos clínicos complejos, éstos son presentados en las sesiones clínicas del Servicio, como si de un paciente del propio hospital se tratase.

2. Cuando se requiere una exploración complementaria de mayor complejidad técnica, (TAC, RMN, etc.) el paciente es remitido al servicio correspondiente del hospital, siendo los resultados enviados a la consulta del Centro.

3. Cuando el paciente requiere cirugía mayor, es dirigido directamente para intervención, es intervenido por el propio especialista del Centro y al Alta, es citado en el Centro para la evaluación de resultados, sin pasar en ningún momento por la consulta del hospital.

En nuestro modelo funcional salvamos muchos de los pasos del peregrinaje de los enfermos: el paciente se remite desde Atención Primaria con una CDD, y en solo 1 ó 2 visitas con el especialista, puede ser indicada la intervención. El establecimiento de este modelo constituye un elemento princeps en la eficiencia de los procesos 
asistenciales, pero requiere una adaptación de todos los estamentos sanitarios, transformando nuestra forma de actuar, planificar y gestionar. Así, es necesario que unidades de apoyo al diagnostico como Radiología estén abiertas a la atención de pacientes "en el día" y que los propios urólogos se apliquen en la realización de exploraciones como la ecografia o la urodinámica. En la cirugía ambulatoria hemos aplicado una Vía Clínica en el propio Centro, de manera que ya en la primera visita el paciente conoce la fecha de intervención; en el caso de cirugía mayor se establecen unos circuitos con el Hospital de Sagunto, según el esquema de la Figura 2.

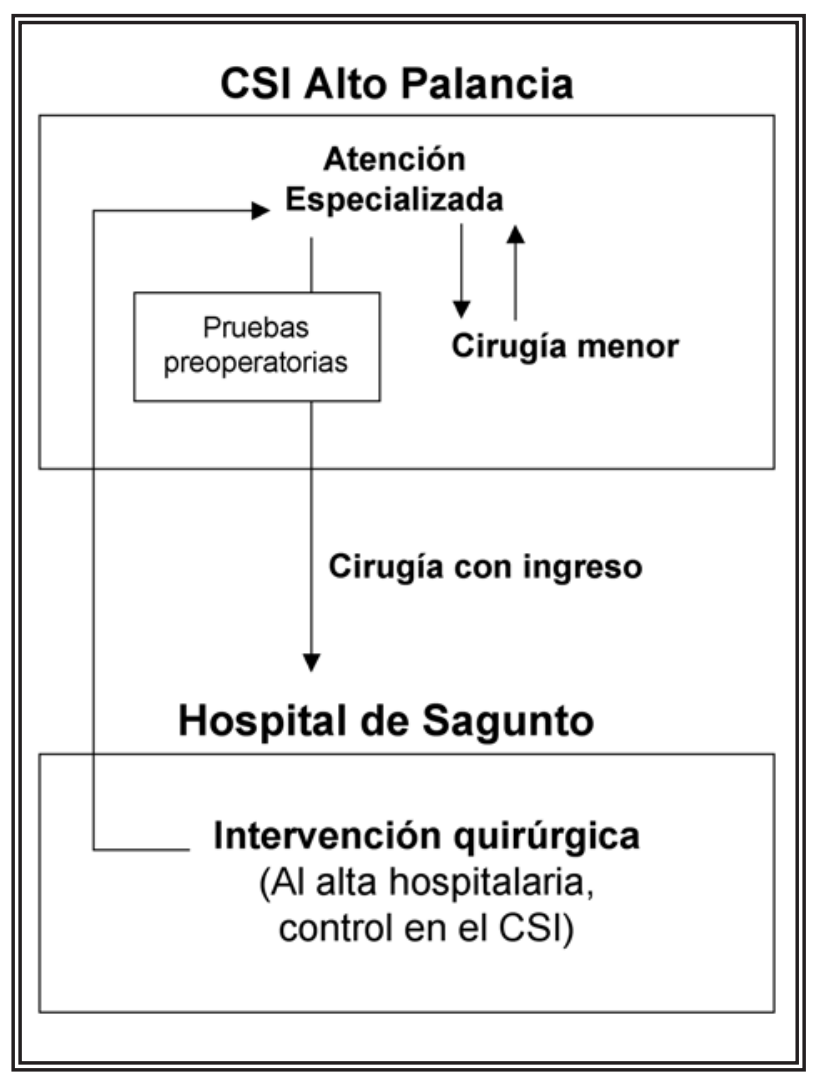

FIGURA 2. Vías Clínicas en el paciente sometido a intervención quirúrgica.

La puesta en marcha de este plan estratégico de asistencia tuvo el apoyo y compromiso de la dirección gestora del Centro y del Hospital de Sagunto, así como del jefe de servicio. Pasamos de una práctica basada en tareas a una práctica basada en resultados: el facultativo especialista controla todo el proceso asistencial hasta el final.
Ventajas del nuevo Modelo Asistencial:

1. Para pacientes atendidos en el Centro. En primer lugar se produce un acercamiento de la asistencia especializada a la población. Disminuye el numero de consultas necesarias para establecer el diagnóstico y tratamiento, pasando de 3 teóricas visitas (una primera al especialista, una segunda para exploraciones complementarias y una tercera para su valoración) a un solo proceso asistencial, reduciéndose los tiempos de demora y masificación de las consultas e incrementando la calidad percibida por el usuario. Además, la Atención Especializada se desarrolla en un entorno conocido, el de Atención Primaria, que contribuye a que el paciente adquiera mayor empatía con el especialista, evidenciándose una continuidad asistencial. Al final el paciente percibe mayor calidad asistencial, si atendemos a las encuestas de satisfacción del Centro.

2. Para los facultativos del Centro. El especialista ve reducida su carga asistencial, trata casos más complejos y puede realizar otras labores como la formativa e investigadora. En nuestro caso los facultativos que acuden al Centro, lo hacen gustosamente, a pesar de que ello supone desplazarse a una mayor distancia respecto del Hospital de Sagunto. Ello se debe a que se desarrolla una actividad eficiente, en un ambiente laboral agradable. El facultativo de Atención Primaria ve realizada una práctica de la medicina más gratificante, al disponer de mayores conocimientos, medios y el apoyo constante del especialista, al que tiene fácil acceso.

3. Para el Sistema Sanitario/Hospital de Referencia: mejora la calidad asistencial, ahorrando en actividad hospitalaria (disminuye la presión asistencial hospitalaria, tanto para primeras visitas como en segundas o sucesivas, así como el tiempo de demora para cirugía menor y exploraciones complementarias). Esto determina también un incremento del nivel de satisfacción para el personal sanitario y pacientes atendidos en el hospital.

\section{CONCLUSIONES}

La asistencia urológica en el marco del Modelo Innovador de Atención Integral permite diagnosticar, orientar y establecer la estrategia terapéutica de un enfermo en un único tiempo asistencial 
(Consulta Única). La interrelación entre niveles asistenciales con visión conjunta de trabajo permite incrementar la calidad percibida por el usuario. Las evidentes ventajas del modelo de CS Integral, tanto para el Usuario como para la Organización Sanitaria deberían orientar las futuras actitudes de los gestores en la Planificación Sanitaria. En la especialidad de Urología se puede aplicar satisfactoriamente el modelo de Consulta Única y las Vías Clínicas con el Hospital de Referencia.

\section{Agradecimientos}

Al Personal del Centro Sanitario Integral "Alto Palancia", que de forma anónima ha colaborado en la consecución de estos resultados y al Consejo Editorial de la Revista "Todo Hospital" por el permiso concedido para la publicación de parte del contenido de este artículo.

\section{REFERENCIAS}

1. Padilla M, Marchal C, Caballero J, García FJ, Lucas IM. Coordinación atención primaria y atención especializada en HBP. En: Del Llano J editor. Medicina basada en la evidencia y la práctica clínica en urología. Jarpyo Editores SA, Madrid 2002;205-216.

2. Plaza V, Rodríguez G, Peiró M, et al. Grado de satisfacción de médicos y pacientes en atención primaria con un nuevo esquema asistencial neumológico. Arch Bronconeumol 2003;39:57-61.

3. Zambrana JL, Jiménez-Ojeda B, Marín M, Almazán S. La consulta única o de alta resolución como una alternativa de eficiencia a las consultas externas hospitalarias tradicionales. Med Clin (Barc) 2002;118:302-305.

4. Ruiz JL, Antonio J, Villalón J, Álvarez JA. La consulta única, como instrumento de mejora de la calidad asistencial, en cirugía ortopédica y traumatología. Todo Hospital 2001; 177:375379.

Dr. J.A. Queipo Zaragozá.

Calle Santa Teresa № 4 - $\mathrm{P}^{\mathrm{a}} 8$.

46530 Puzol (Valencia)

E-mail: queipo@pulso.com

(Trabajo recibido el 3 noviembre de 2004) 